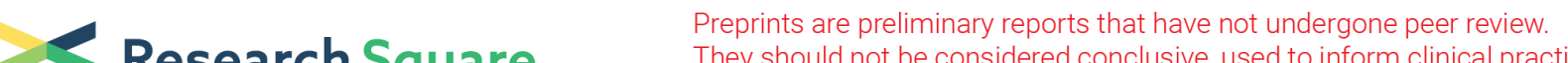 $\begin{array}{ll}\text { Research Square } & \text { They should not be considered conclusive, used to inform clinical practice, } \\ \text { or referenced by the media as validated information. }\end{array}$
}

\section{MiR-574-5P, miR-1827, And miR-4429 As Potential Biomarkers For Schizophrenia}

\section{Omran Davarinejad}

Kermanshah University of Medical Sciences

\section{Sajad Najafi}

Shahid Beheshti University of Medical Sciences School of Nursing and Midwifery

Hossein Zhaleh

Kermanshah University of Medical Sciences

\section{Farzaneh Golmohammadi}

Kerman University of Medical Sciences

\section{Farnaz Radmehr}

Kermanshah University of Medical Sciences

\section{Mostafa Alikhani}

Kerman University of Medical Sciences

\section{Reza Heidari Moghadam}

Kerman University of Medical Sciences

Yazdan Rahmati ( $\square$ yazdanrahmati68@gmail.com )

Kermanshah University of Medical Sciences https://orcid.org/0000-0001-9257-9395

\section{Research Article}

Keywords: Schizophrenia, metaDE, WGCNA, microRNA, Real-Time PCR

Posted Date: September 2nd, 2021

DOl: https://doi.org/10.21203/rs.3.rs-844121/v1

License: (c) (1) This work is licensed under a Creative Commons Attribution 4.0 International License. Read Full License

Version of Record: A version of this preprint was published at Journal of Molecular Neuroscience on November 22nd, 2021. See the published version at https://doi.org/10.1007/s12031-021-01945-0. 


\section{Abstract}

Schizophrenia is a severe chronic debilitating disorder with millions of affected individuals. Lack of a reliable mollecular diagnostic invokes the identification of novel biomarkers. To elucidate the molecular basis of the disease, two mRNA expression arrays including GSE93987 and GSE38485, and one miRNA array, GSE54914, were downloaded from GEO, and meta-analysis was performed for mRNA expression arrays by employment of metaDE package. By WGCNA package, we performed network analysis for both mRNA expression arrays separately. Then, we made protein-protein interaction network for significant modules. Limma package was employed to analyze the miRNA array and dysregulated miRNAs (DEMs) were identified. Using genes of significant modules and DEMs, a mRNA-miRNA network was constructed and hub genes and miRNAs were identified. To confirm the dysregulation of genes, expression values were evaluated by available datasets including GEO series GSE62333, GSE93987, and GSE38485. The ability of the detected hub miRNAs to discriminate Schizophrenia from healthy controls was evaluated by assessing the receiver-operating curve. Finally, by performing Real-Time PCR, the expression level of genes and miRNAs were evaluated in 40 Schizophrenia patients compared with healthy controls. The results confirmed dysregulation of hsa-miR-574-5P, hsa-miR-1827, hsa-miR-4429, CREBRF, ARPP19, TGFBR2, and YWHAZ in blood samples of schizophrenia patients.

\section{Introduction}

Schizophrenia (SCZ) is a chronic debilitating mental disease with a complex identity considered as one of the most mysterious human disorders (1). It mainly appears during adulthood primary years affecting both genders, any race, and genetic backgrounds. According to the global estimations, about 20 million people are living with SCZ around the world (2). The affected patients are expected to have a reduced lifespan of 12-15 years compared to healthy individuals which shows higher mortality compared to physical diseases (3). SCZ diagnosis is based on the psychopathological features including psychosis, hallucination or delusion, and impaired emotions and volition in absence of any medical disorder (4). Thus, diagnosis is made when the clinical manifestations of the disease have appeared and any diagnostic approach to screen the individuals at risk is of special importance. The impairment of neurotransmitters like dopamine and gamma aminobutyric acid (GABA) has been the center to the main therapeutical strategies in SCZ, however results of current treatments are not satisfying indicate requirement to new concepts. Although etiology of SCZ is still unknown, we already have known that individuals who have a history of SCZ in their first-grade family members have a higher risk of the disorder. Furthermore, an accumulating evidence suggest familial predisposition as the main risk factor for the disease in addition to other provoking conditions like pregnancy and simultaneous infections, impairments in neurological development and cannabis abuse are other risk factors for SCZ (5). Early diagnosis may help improve the prognosis through enhancing therapeutical responses to early interventions, prevention of rapid progression of the disease and related social disability, providing earlier consultation for the affected families and reducing later frequencies. Development of any molecular 
diagnostic approach for SCZ requires more detailed unveiling the mechanisms responsible for pathogenesis in the disease.

MicroRNAs (miRNAs) are a novel group of non-coding RNAs (ncRNAs) with an average length of 22 nucleotides which don't encode any protein unlike known class of messenger RNAs (mRNAs). Their functions have not been completely understood, however key regulatory roles on eukaryotic gene expression described for an increasing number of identified miRNAs. In a variety of biological phenomena such as stress responses (6), cell proliferation, differentiation, and death (7), embryogenesis, organ development and function $(8,9)$, miRNAs have been recognized with regulatory functions in multicellular organisms. They mainly act at the post-transcriptional level in interaction with protein complexes via binding to complementary regions mostly located on the $3 \otimes$ untranslated region (UTR) of the target mRNA and thus, drive their degradation by RNA-induced silencing complex (RISC) leading to repression of the expression of critical proteins which play role in essential cellular processes. As expected, biogenesis, processing, nucleolar export, and stability of miRNAs is faced to precise regulation (10) which also, suggests their substantial roles. Continuously growing evidence have demonstrated association of aberrant expression of miRNAs and numerous human diseases including diabetes (11), osteoporosis (12), neurodegenerative diseases (13), viral infections (14), and various types of cancer (15). Several miRNAs, also have been shown to aberrantly expressed in serum samples (16), cortex tissues (17), and gene analyses of SCZ patients (18). As a result, differentially expressed miRNAs can be considered as potential SCZ biomarkers (19) which are easily detected in blood samples of the patients and eventually used in early diagnosis.

Through employment of bioinformatics tools like Weighted Gene Co-Expression Network Analysis (WGCNA) in several studies, interactions between risk co-expressed gene have been identified in patients with SCZ $(20,21)$ in addition to normal and other mental diseases $(22)$. For instance, Wen et al. (23) identified 134 SCZ-specific key genes using WGCNA and Radulescu et al. (24) found 12 gene coexpression network modules in postmortem brain samples of SCZ patients. A number of other modules have been discovered using construction of WGCNA in other studies (25-27). To date, multiple susceptibility genes and characteristic genetic changes have been identified in association with susceptibility to SCZ development playing role in neurodevelopment which is believed to be affected in $\mathrm{SCZ}$ and other psychiatric disorders. These genes include immune function-related gene loci on chromosome 6p22.1 (28), chromosome 22q11.2 deletion (29), and alleles of 5-hydroxytryptamine (5-HT) receptor gene, and dopamine D3 receptor gene (30). Studies have shown that thousands of single nucleotide polymorphisms (SNPs) and copy number variants (CNVs) can explain parts of the genetic susceptibility to SCZ (31). Genetic mechanisms, however have not been convincing independently in elucidation of the SCZ pathophysiology (32). Same to many other diseases, epigenetic regulations along with genetic alterations including methylation, acetylation, phosphorylation, and SUMOylation on DNA bases or histone residues have been found to be involved in molecular mechanisms associated with SCZ.

To our knowledge and according to the literature, protein networks and protein-miRNA networks have not been identified in SCZ. In this study, we aimed to identify aberrantly expressed genes and associated 
cellular mechanisms and miRNAs among Iranian SCZ patients using gene co-expression network analyses. Hub miRNAs and genes were verified using quantified reverse transcriptase polymerase chain reaction (qRT-PCR) and their diagnostic values were assessed. The candidate miRNAs and genes can be considered as SCZ biomarkers.

\section{Materials And Methods}

\section{Data collection and processing}

Three expression arrays including Gene Expression Omnibus (GEO) series number GSE93987, GSE38485 and GSE54914 were obtained from GEO database (http://www.ncbi.nlm.nih.gov/geo/). In GSE93987, individual pyramidal cells in dorsolateral prefrontal cortex layers 3 or 5 were captured by laser microdissection from 36 subjects diagnosed with SCZ or schizoaffective disorder and matched healthy controls. In GSE38485, gene co-expression network in whole blood samples retrieve from 106 SCZ patients was compared to those from 96 healthy controls. In GSE54914through a processing method, the probes were converted to the corresponding genes using their platform, then the raw data were normalized by quantile normalization function in Limma package (33).

\section{Meta-analysis}

Using metaDE package (2), meta-analysis for two mentioned mRNA expression datasets was performed and differentially expressed genes (DEGs) between SCZ and healthy samples were identified. A false discovery rate $(F D R)<0.05$ was selected as the significance threshold for screening of the DEGs.

\section{Weighted gene co-expression network construction and module detection}

After identification of DEGs using metaDE package, the corresponded gene co-expression networks were constructed using the WGCNA package (34). In the process of network construction, primarily, a matrix of similarity was made by calculating the correlations of all gene pairs. By applying pickSoftThreshold function, the appropriate soft-thresholding power B was selected to access the scale-free topology. Afterwards, to reduce the effect of noise and spurious associations, adjacency was transformed to topological overlap matrix and the corresponding dissimilarity was calculated. Hierarchical clustering was utilized to produce a dendrogram of genes Significantly associated modules with the measured clinical traits were selected according to two parameters including correlation and $p$-value in the next step. Then, we quantified the association of individual genes with our trait of interest (status) by defining Gene Significance (GS) as the absolute value of the correlation between the gene and the corresponded trait. For each module, we also defined a quantitative measure of module membership (MM) as the correlation of the module eigengene and the gene expression profile. This allows us to quantify the similarity of all genes on the array to every module. In the final step of network analysis using the GS and MM measures, we identified the genes with high significance for status as well as high MM in the selected modules. 


\section{Protein-protein interaction (PPI) network analysis and Pathway enrichment analysis}

To identify the protein-protein interaction (PPI) information, the proteins of the selected modules were matched to the search tool for the retrieval of interacting genes (STRING) database ( https://string$\mathrm{db}$. org/cgi/input.pl). The retrieved data was imported to the Cytoscape software (35) and the corresponding PPI was visualized based on the degree and betweenness centrality. To elucidate potential dysregulated signaling pathways, ClueGO was utilized and the most important signaling pathways were identified (36).

\section{Identification of differentially expressed miRNAs (DEMs)}

By searching the GEO database, GSE54914 miRNA array was used for the identification of differentially expressed miRNAs (DEMs) between SCZ patients and healthy controls. Significant miRNAs were identified based on $p$-value threshold of $<0.05$ and $\log F C>1$.

\section{MiRNA-protein interaction network construction and Identification of hub miRNAs}

DEMs and the most important genes retrieved from PPI were used for the construction of miRNA-protein interaction. For this purpose, CyTargetLinker plugin (37) based on Cytoscape software was employed, and a regulatory network between miRNAs and genes was made using miRBase (38), TargetScan (39), and TransmiR (40). Considering the miRNA-protein network, three miRNAs and three genes were identified for the rest of the analysis.

\section{Validation of detected miRNAs by ROC curve}

We plotted the receiver operating characteristic (ROC) curve to verify the diagnostic performance of core miRNAs based on GSE54914 and estimated the area under the curve (AUC). The miRNAs with $p$-value < 0.05 and $A U C>0.8$ were considered as strong potential diagnostic biomarkers.

\section{Validation of detected genes and miRNAs based on SCZ clinical samples}

To further verify the results of bioinformatics data, we performed quantitative qRT-PCR(qRT-PCR) for evaluation of the expression level of the selected genes and miRNAs in clinical SCZ patient samples ( $\mathrm{n}=$ 40) recruited to Imam Khomeini, Mohammad Kermanshahi and Farabi Hospitals, Kermanshah university of medical sciences, Kermanshah, Iran. Written informed consent was obtained from all patients.

\section{RNA extraction and cDNA production}

Whole blood RNA purification kit (Qiagen Cat No. 52304) was used for extraction of RNA. Total RNA was extracted from $2 \mathrm{ml}$ whole blood collected from each subject. Using reverse transcriptase enzyme, complementary DNA (cDNA) was synthesized, and then treated with DNase I enzyme to remove the genomic DNA. The purity of the extracted RNA was performed by UV-spectrophotometry using the absorbance ratio at $260 \mathrm{~nm} / 280 \mathrm{~nm}$. 


\section{Primer design}

The online miRNA designing tool (http://genomics.dote.hu:8080/mirnadesigntool/) was employed for primer designation. For the expression analysis of the selected genes, primer design was performed using GenScript online tool (https://www.genscript.com/tools/real-time-pcr-tagman-primer-design-tool).

\section{qRT-PCR}

Primers arrived in the lyophilized form (Cinaclone, Iran). For preparation, sterile distilled water was added to each tube containing lyophilized primer (based on the data for each primer), and the solution was placed in stoke at $-20^{\circ} \mathrm{C}$. Applied Biosystems Real-Time PCR instrument and Takara SYBR green kit were used for the analysis of miRNA and mRNA expression changes. We also set up for each gene at each time, a negative control to examine the presence of contamination in each reaction. The expression fold change of genes examined in this study was evaluated using the Threshold Cycle (CT) method by the formulas below. $5 s$ rRNA and GAPDH were used as the reference genes for this study.

$\mathrm{R}=2^{-(\Delta \Delta \mathrm{CT})}$

$\Delta \Delta \mathrm{CT}=\left(\mathrm{CT}_{\text {target }}-\mathrm{CT}_{\text {reference }}\right)$ healthy ${ }^{-(\mathrm{CT}}$ target $^{-\mathrm{CT}}$ reference $)$ patient

\section{Results}

\section{Meta-analysis}

Two datasets with available mRNA expression data for SCZ including GSE93987 and GSE38485 and also one miRNA expression data, GSE54914, were downloaded from GEO database. After performing meta-analysis by the metaDE package for mRNA expression arrays, 3840 DEGs were identified and selected for further investigations (Figure 1).

\section{Construction of Weighted Gene Co-Expression Network}

WGCNA package was used to construct the co-expressed networks and identify the co-expression modules for results of the meta-analysis of each mRNA expression data separately. Clustering dendrogram of samples for DEGs of each dataset was performed based on their Euclidean distance (Figure 2a and 3a). We then quantified the association of individual genes with the trait of interest (SCZ and healthy) by defining the GS and MM values allowing us to quantify the similarity of all genes on the array to every module (Figure $2 \mathrm{~b}$ and $3 \mathrm{~b}$ ). A total number of 43 modules were identified for the DEGs of GSE38485, and only genes of the lightcyan module were selected for the rest of the analysis. Seven modules were identified for GSE93987 and blue module was selected for the rest of the analysis (figure $3 b)$. It is obvious that GS and MM measures are highly correlated, illustrating that genes significantly associated with a trait are often also the most important (central) elements of modules associated with the trait (Figure $2 \mathrm{c}$ and $3 \mathrm{c}$ ). Based on figure $2 \mathrm{c}$, genes of light cyan module show correlation and $p$-value 
of 0.63 and:3.5e-24, respectively and according to the figure $3 c$, genes of blue module demonstrate the corresponding values of 0.32 and $4.6 \mathrm{e}-10$, respectively.

\section{Protein-protein interaction network and pathway enrichment analysis}

All genes of favorite modules (lightcyan and blue) were used for network construction. For lightcyan module, 242 genes were selected and using both of STRING database and Cytoscape software, the PPI network was made (Figure 4a). The color and size of each protein were set based on degree and betweenness centrality. For the blue module, 362 proteins were selected, and according to the same protocol, the corresponding protein network was constructed (Figure 5a). To pathway enrichment analysis, the CluGO plugin based on Cytoscape software was used to illustrate the results of KEGG path analysis for each of the gene groups separately (Figure $4 b$ and $5 b$ ). For genes of lightcyan module, some dysregulated signaling pathways included Vitamin B6 binding, fucosyltransferase activity, single stranded DNA binding, polyubiquitin modification-dependent protein binding, DNA replication origin binding, and double stranded RNA binding. Also, several signaling pathways were identified to be dysregulated for the blue module which included epithelial cell signaling in helicobacter pylori infection, vasopressin-regulated water reabsorption, dopaminergic synapse, proteasome, and salmonella infection.

\section{Identification of differentially expressed miRNAs}

In GSE54914, the miRNA expression of 18 SCZ and 12 controls compared with each other. Raw data were normalized by log2 transformation and also quantile normalization function in Limma package. The data unveiled 191 upregulated miRNAs and 2 downregulated miRNAs (Figure 6).

\section{MRNA-miRNA network construction}

Based on two initial mRNA arrays, two miRNA-mRNA networks were made (Figure 6 and Figure 7). According to the DEGs extracted from GSE38485 and DEMs identified by GSE54914, a network was made (figure 6). As obvious, hsa-miR-4429 shows the direct effect on some DEGs including SORCS1, ZNF652, CHEK1, FAM63B, PDCD4, and FOXM1. Hsa-miR-574-5P demonstrates direct regulatory effect on MCM8, SLC35E1, HS6ST3, and ZRANB. Hsa-miR-1827 regulates the expression of some DEGs like ACBD7, XPNPEP3, PPM1K, and CENPM.

\section{Identification of hub genes}

Using DEGs extracted from GSE93987 and DEMs identified by GSE54914, a miRNA-mRNA network was made (figure 7). According to this network, three miRNAs including hsa-miR-4429, hsa-miR-1827, and hsamiR-574-5P play a central role. Therefore, we considered these three as the hub miRNAs for further analyses. The common target genes of these miRNAs were identified using Cytargetlinker plugin based on Cytoscape software (Figure 8). Several genes like CREBRF, ARPP19, TGFBR2, YWHAZ, SRSF7, MAPK1IP1L, FAM117B, and BVES were demonstrated to be regulated by the hub miRNAs. We also 
evaluate the expression value of detected genes in three independent datasets including GSE62333, GSE93987, and GSE38485 (Figure9).

\section{Validation of miRNAs by receiver-operating characteristic (ROC) curve}

The ROC curves constructed for raw data of GSE54914 using a single-gene test in SCZ versus normal tissues for three miRNAs by plotting sensitivity versus specificity. The results demonstrated significant values for the hub miRNAs. These included for hsa-miR-4429 (AUC: 0.9 and $p$-value: 0.0001), hsa-miR1827 (AUC: 0.84 and $p$-value: 0.0013), and hsa-miR-574-5P (AUC: 0.9 and $p$-value: 0.0002). The AUC was analyzed by the Hanley and McNeil method (Figure 10).

\section{qRT-PCR}

To evaluate the expression level of the hub genes and miRNAs, qRT-PCR was carried out in 40 paired clinical samples (Figure 11). Among the selected genes, CREBRF, TGFBR2, YWHAZ, and MAPK1IP1L demonstrated downregulation in SCZ samples compared to healthy controls, while the hub miRNAs were shown to be upregulated.

\section{Discussion}

SCZ is one of the most debilitating mental disorders and a complex disease with unknown etiology. Diagnosis is made based on the clinical manifestations on appearance which makes the disease hardly manageable. Real time diagnosis may help improve or slow down progression of the consequences and response to treatment which eventually promote the patient's quality of life and social activities. Molecular diagnostics are potential nominates for on time detection of susceptible or affected individuals. In accordance to the progressions in the past decades, genetic studies have shown correlation between several genes and susceptibility to SCZ development in people with familial history. Transcriptome studies, also have demonstrated dysregulation of a growing number of miRNAs in tissues and plasma of SCZ patients. MiRNAs as a novel group of ncRNAs act as key regulators of gene expression playing role in critical biological processes. Dysregulation in their biogenesis has been associated with various human diseases including SCZ for which a number of miRNAs have been identified to be aberrantly expressed in patient tissues compared to healthy individuals. MiRNAs are easily detected in human body fluids suggesting them as potential biomarkers for a wide number of disorders particularly for those without diagnostic approaches or diagnosed via invasive methods. Since accessibility to brain biopsies is not possible for screening of SCZ susceptible individuals, development of a diagnostic approach based on miRNAs potentially can benefit this society.

In the current study, via network construction we identified 3 hub miRNAs including hsa-miR-574-5P, hsamiR-1827, and hsa-miR-4429 to be aberrantly expressed in blood sample of SCZ patients confirmed by qRT-PCR results. These miRNAs demonstrated acceptable diagnostic values in ROC curve. Hsa-miR-5745P, also known as miR-574-5P, has been studied as cancer biomarker for several human tumors like lung (41), colorectal (42), and squamous cell carcinoma (43). It is also known to be affected in other 
conditions such as rheumatoid arthritis (44), and diabetes mellitus (45). Other hub miRNAs including hsamiR-1827, and hsa-miR-4429 have been associated with particular various types of cancers. Mutations in any of the up-regulated genes have been correlated with various human conditions mainly with cancers and not associated with mental disorders. Thus, association of these genes with SCZ pathogenesis can be further explored to find diagnostic and therapeutical targets.

Overall, identification of these miRNAs and genes, already have been associated with particularly cancer, in blood samples of SCZ patients may help finding a diagnostic approach which make them possible to be easily detected in peripheral blood and so, earlier screening and monitoring the affected individuals with SCZ can be achieved. Further investigations are recommended for potential clinical application of these miRNAs.

In conclusion, this study is one of the first attempts to construct a miRNA-mRNA network using metaDE and WGCNA and limma packages. We identified 4 hub genes and 3 miRNAs in three separate datasets. Then their expression was verified by other independent dataset and real-time PCR, and their prognosis and diagnosis power validated by ROC curve.

\section{Declarations}

\section{Acknowledgments}

The authors would like to thank the clinical Research Development Unit (CRDU) of Imam Khomeini, Mohammad Kermanshahi and Farabi Hospital, Kermanshah university of medical sciences, Kermanshah, Iran for their support, cooperation and assistance throughout the period of study (Grant number: 97542).

\section{Funding Source}

This article is the result of the findings of the research project 97542that was approved and financed by Vice Chancellor for research and Research and Technology, Kermanshah University of Medical Sciences, Kermanshah, Iran

\section{Ethics Approval and Consent to Participate}

All patients gave their signed written informed consent letters. Medical Research and Ethical Committee of Kermanshah university of Medical Sciences (Kermanshah, Iran; registration No.

IR.KUMS.REC.1397.490; grant number 97542) approved the study performed under ethical principles contained in the $7^{\text {th }}$ and current (2013) editions of Helsinki Declaration.

\section{Availability of data and materials}

The data used to support the findings of this study are available from the corresponding author upon request. 


\section{Authors' contributions}

Conceptualization: Yazdan Rahmati and Omran Davarinejad, Computational analyses and Experiments: Yazdan Rahmati, Sajad Najafi, and Hossein Zhaleh, Writing manuscript: Yazdan Rahmati, Sajad Najafi, Farzaneh Golmohammadia, Farnaz Radmehra, Mostafa Alikhania, Reza Heidari Moghadam, Supervision and Finalization: Yazdan Rahmati

\section{Authors' information}

Clinical Research Development Center, Imam Khomeini, and Mohammad Kermanshahi and FarabiHospitals, Kermanshah University of Medical Sciences, Kermanshah, Iran

\section{Omran Davarinejad, Farzaneh Golmohammadi, Farnaz Radmehra, Mostafa Alikhania, Yazdan Rahmati}

Student Research Committee, Department of Medical Biotechnology, School of Advanced Technologies in Medicine, Shahid Beheshti University of Medical Sciences, Tehran, Iran

\section{Sajad Najafi}

Substance Abuse Prevention Research Center, Health Institute, Kermanshah University of Medical Sciences, Kermanshah, Iran

\section{Hossein Zhaleh,}

Cardiovascular Research Center, Health Institute, Kermanshah University of medical sciences, Kermanshah, Iran

\section{Reza Heidari Moghadamd}

\section{References}

1. van Os J, Kapur S. Schizophrenia. Lancet. 2009;374(9690):635-45.

2. James SL, Abate D, Abate KH, Abay SM, Abbafati C, Abbasi N, et al. Global, regional, and national incidence, prevalence, and years lived with disability for 354 diseases and injuries for 195 countries and territories, 1990\&\#x2013;2017: a systematic analysis for the Global Burden of Disease Study 2017. The Lancet. 2018;392(10159):1789-858.

3. van Os J, Kapur S. Schizophrenia. The Lancet. 2009;374(9690):635-45.

4. Taylor MA, Gaztanaga P, Abrams R. Manic-depressive illness and acute schizophrenia: a clinical, family history, and treatment-response study. Am J Psychiatry. 1974;131(6):678-82.

5. Mäki P, Veijola J, Jones PB, Murray GK, Koponen H, Tienari P, et al. Predictors of schizophrenia-a review. British Medical Bulletin. 2005;73-74(1):1-15. 
6. Leung AKL, Sharp PA. MicroRNA Functions in Stress Responses. Molecular Cell. 2010;40(2):205-15.

7. Bushati N, Cohen SM. microRNA Functions. Annual Review of Cell and Developmental Biology. 2007;23(1):175-205.

8. Kloosterman WP, Plasterk RH. The diverse functions of microRNAs in animal development and disease. Developmental cell. 2006;11(4):441-50.

9. Maes OC, An J, Sarojini H, Wang E. Murine microRNAs implicated in liver functions and aging process. Mechanisms of Ageing and Development. 2008;129(9):534-41.

10. Treiber T, Treiber N, Meister G. Regulation of microRNA biogenesis and function. Thromb Haemost. 2012;107(04):605-10.

11. Tang X, Tang G, Özcan S. Role of microRNAs in diabetes. Biochimica Et Biophysica Acta (BBA)-Gene Regulatory Mechanisms. 2008;1779(11):697-701.

12. Van Wijnen AJ, Van De Peppel J, Van Leeuwen JP, Lian JB, Stein GS, Westendorf JJ, et al. MicroRNA functions in osteogenesis and dysfunctions in osteoporosis. Current osteoporosis reports. 2013;11(2):7282.

13. Nelson PT, Wang WX, Rajeev BW. MicroRNAs (miRNAs) in neurodegenerative diseases. Brain pathology. 2008;18(1):130-8.

14. Islam MS, Khan MAAK, Murad MW, Karim M, Islam ABMMK. In silico analysis revealed Zika virus miRNAs associated with viral pathogenesis through alteration of host genes involved in immune response and neurological functions. Journal of medical virology. 2019;91(9):1584-94.

15. Seven M, Karatas OF, Duz MB, Ozen M. The role of miRNAs in cancer: from pathogenesis to therapeutic implications. Future Oncology. 2014;10(6):1027-48.

16. Shi W, Du J, Qi Y, Liang G, Wang T, Li S, et al. Aberrant expression of serum miRNAs in schizophrenia. Journal of Psychiatric Research. 2012;46(2):198-204.

17. Perkins DO, Jeffries CD, Jarskog LF, Thomson JM, Woods K, Newman MA, et al. microRNA expression in the prefrontal cortex of individuals with schizophrenia and schizoaffective disorder. Genome Biology. 2007;8(2):R27.

18. Hauberg ME, Roussos P, Grove J, Børglum AD, Mattheisen M, Consortium ftSWGotPG. Analyzing the Role of MicroRNAs in Schizophrenia in the Context of Common Genetic Risk Variants. JAMA Psychiatry. 2016;73(4):369-77.

19. He K, Guo C, He L, Shi Y. MiRNAs of peripheral blood as the biomarker of schizophrenia. Hereditas. 2017;155(1):9. 
20. Torkamani A, Dean B, Schork NJ, Thomas EA. Coexpression network analysis of neural tissue reveals perturbations in developmental processes in schizophrenia. Genome Res. 2010;20(4):403-12.

21. Kim Y, Giusti-Rodriguez P, Crowley JJ, Bryois J, Nonneman RJ, Ryan AK, et al. Comparative genomic evidence for the involvement of schizophrenia risk genes in antipsychotic effects. Molecular Psychiatry. 2018;23(3):708-12.

22. Oldham MC, Konopka G, Iwamoto K, Langfelder P, Kato T, Horvath S, et al. Functional organization of the transcriptome in human brain. Nat Neurosci. 2008;11(11):1271-82.

23. Wen Y-D, Xia Z-W, Li D-J, Cheng Q, Zhao Q, Cao H. Genetic Profiles Playing Opposite Roles of Pathogenesis in Schizophrenia and Glioma. Journal of Oncology. 2020;2020:3656841.

24. Radulescu E, Jaffe AE, Straub RE, Chen Q, Shin JH, Hyde TM, et al. Identification and prioritization of gene sets associated with schizophrenia risk by co-expression network analysis in human brain. Molecular Psychiatry. 2020;25(4):791-804.

25. Liu L, Zhao J, Chen Y, Feng R. Metabolomics strategy assisted by transcriptomics analysis to identify biomarkers associated with schizophrenia. Analytica Chimica Acta. 2020;1140:18-29.

26. Zhang Y, You X, Li S, Long Q, Zhu Y, Teng Z, et al. Peripheral Blood Leukocyte RNA-Seq Identifies a Set of Genes Related to Abnormal Psychomotor Behavior Characteristics in Patients with Schizophrenia. Medical science monitor : international medical journal of experimental and clinical research. 2020;26:e922426-e.

27. Feltrin ASA, Tahira AC, Simões SN, Brentani H, Martins DC, Jr. Assessment of complementarity of WGCNA and NERI results for identification of modules associated to schizophrenia spectrum disorders. PloS one. 2019;14(1):e0210431-e.

28. Purcell SM, Wray NR, Stone JL, Visscher PM, O'Donovan MC, Sullivan PF, et al. Common polygenic variation contributes to risk of schizophrenia and bipolar disorder. Nature. 2009;460(7256):748-52.

29. Bassett AS, Chow EW, Weksberg R, Brzustowicz L. Schizophrenia and genetics: new insights. Current psychiatry reports. 2002;4(4):307-14.

30. O'Donovan MC, Owen MJ. Candidate-gene association studies of schizophrenia. American journal of human genetics. 1999;65(3):587-92.

31. Maric NP, Svrakic DM. Why schizophrenia genetics needs epigenetics: a review. Psychiatria Danubina. 2012;24(1):2-18.

32. Pries L-K, Gülöksüz S, Kenis G. DNA Methylation in Schizophrenia. In: Delgado-Morales R, editor. Neuroepigenomics in Aging and Disease. Cham: Springer International Publishing; 2017. p. 211-36. 
33. Smyth GK. Limma: linear models for microarray data. Bioinformatics and computational biology solutions using R and Bioconductor: Springer; 2005. p. 397-420.

34. Langfelder P, Horvath S. WGCNA: an R package for weighted correlation network analysis. BMC bioinformatics. 2008;9(1):1-13.

35. Shannon P, Markiel A, Ozier O, Baliga NS, Wang JT, Ramage D, et al. Cytoscape: a software environment for integrated models of biomolecular interaction networks. Genome research. 2003;13(11):2498-504.

36. Bindea G, Mlecnik B, Hackl H, Charoentong P, Tosolini M, Kirilovsky A, et al. ClueGO: a Cytoscape plugin to decipher functionally grouped gene ontology and pathway annotation networks. Bioinformatics. 2009;25(8):1091-3.

37. Kutmon M, Kelder T, Mandaviya P, Evelo CT, Coort SL. CyTargetLinker: a cytoscape app to integrate regulatory interactions in network analysis. PloS one. 2013;8(12):e82160.

38. Griffiths-Jones S, Saini HK, Van Dongen S, Enright AJ. miRBase: tools for microRNA genomics. Nucleic acids research. 2007;36(suppl_1):D154-D8.

39. Agarwal V, Bell GW, Nam J-W, Bartel DP. Predicting effective microRNA target sites in mammalian mRNAs. elife. 2015;4:e05005.

40. Wang J, Lu M, Qiu C, Cui Q. TransmiR: a transcription factor-microRNA regulation database. Nucleic acids research. 2010;38(suppl_1):D119-D22.

41. Foss KM, Sima C, Ugolini D, Neri M, Allen KE, Weiss GJ. miR-1254 and miR-574-5p: serum-based microRNA biomarkers for early-stage non-small cell lung cancer. Journal of thoracic oncology. 2011;6(3):482-8.

42. Cui Z, Tang J, Chen J, Wang Z. Hsa-miR-574-5p negatively regulates MACC-1 expression to suppress colorectal cancer liver metastasis. Cancer cell international. 2014;14(1):1-9.

43. Yang M, Liu R, Sheng J, Liao J, Wang Y, Pan E, et al. Differential expression profiles of microRNAs as potential biomarkers for the early diagnosis of esophageal squamous cell carcinoma. Oncology reports. 2013;29(1):169-76.

44. Hegewald AB, Breitwieser K, Ottinger SM, Mobarrez F, Korotkova M, Rethi B, et al. Extracellular miR574-5p Induces Osteoclast Differentiation via TLR 7/8 in Rheumatoid Arthritis. Front Immunol. 2020;11:585282.

45. Wang F, Li Z, Zhao M, Ye W, Wu H, Liao Q, et al. Circulating miRNAs miR-574-5p and miR-3135b are potential metabolic regulators for serum lipids and blood glucose in gestational diabetes mellitus. Gynecological Endocrinology. 2021;37(7):665-71. 


\section{Figures}

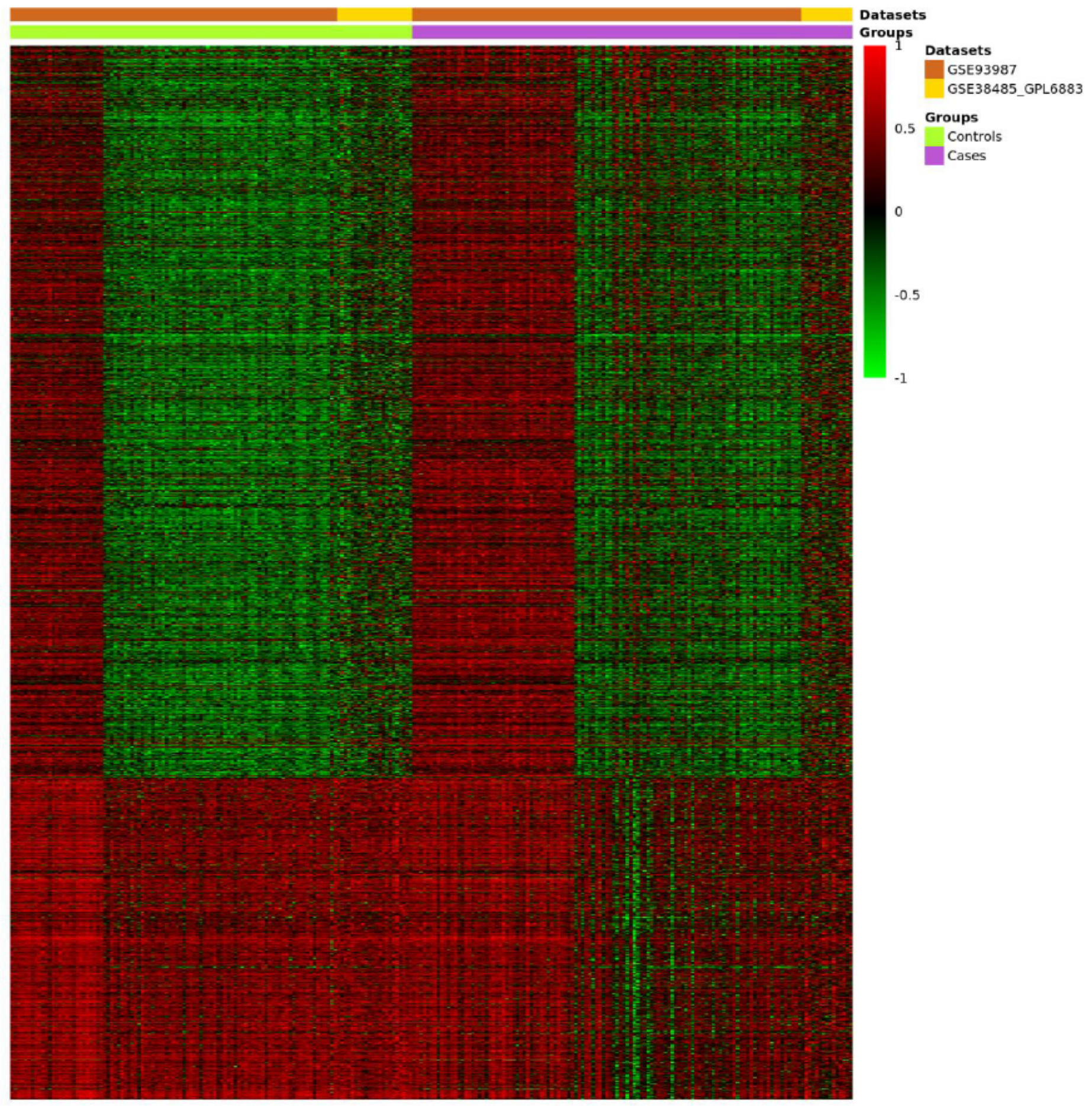

\section{Figure 1}

heatmap for DEGs between schizophrenia and healthy controls for two datasets including GSE93987 and GSE38485. red colors represents upregulated genes and green colors represents downregulated genes 

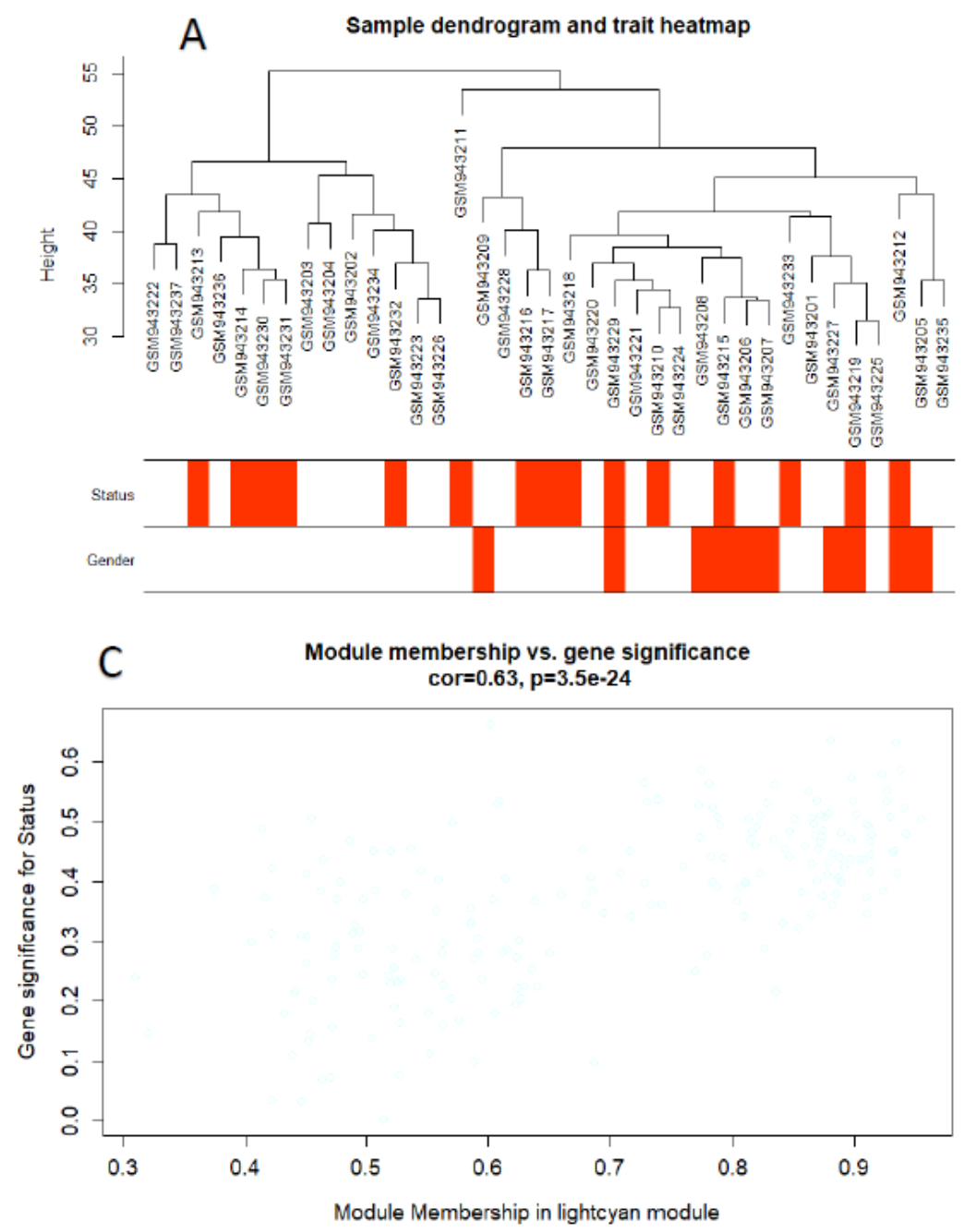

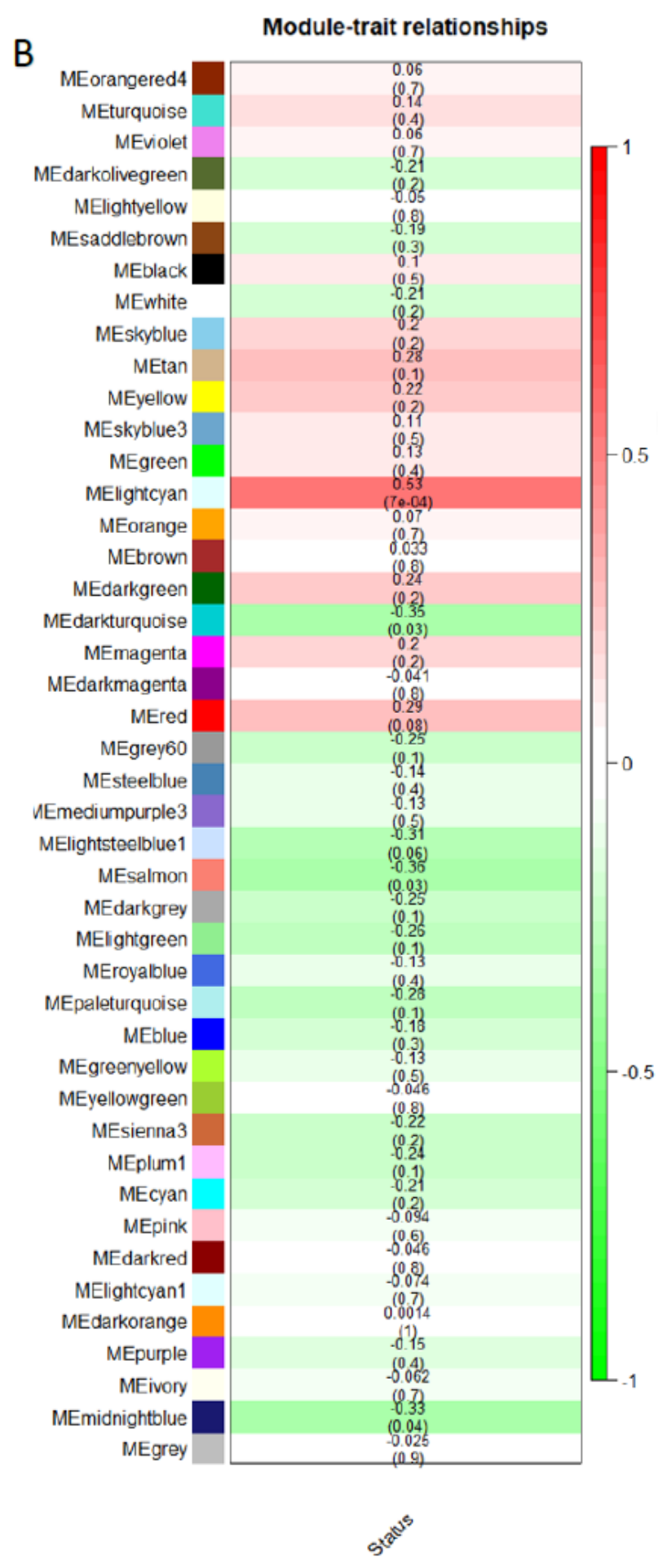

\section{Figure 2}

Network construction for GSE38485 using WGCNA. (A) Clustering dendrogram of samples based on their Euclidean distance. (B) Module-trait associations. Each row corresponds to a module eigengene, column to a trait. Each cell contains the corresponding correlation and p-value. The table is color-coded by correlation according to the color legend. (C) A scatterplot of Gene Significance (GS) for weight vs. Module Membership (MM) in the lightcyan module. 

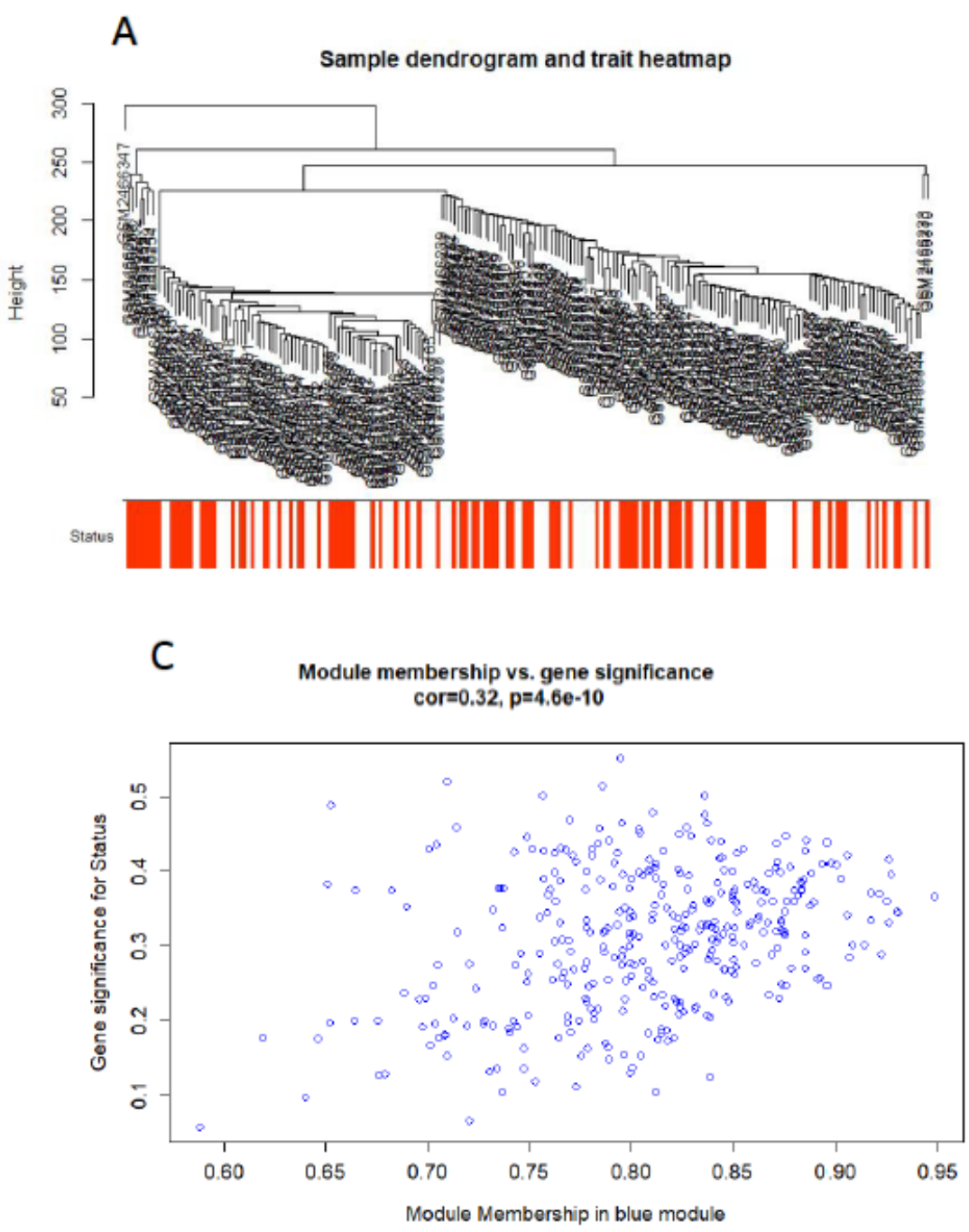

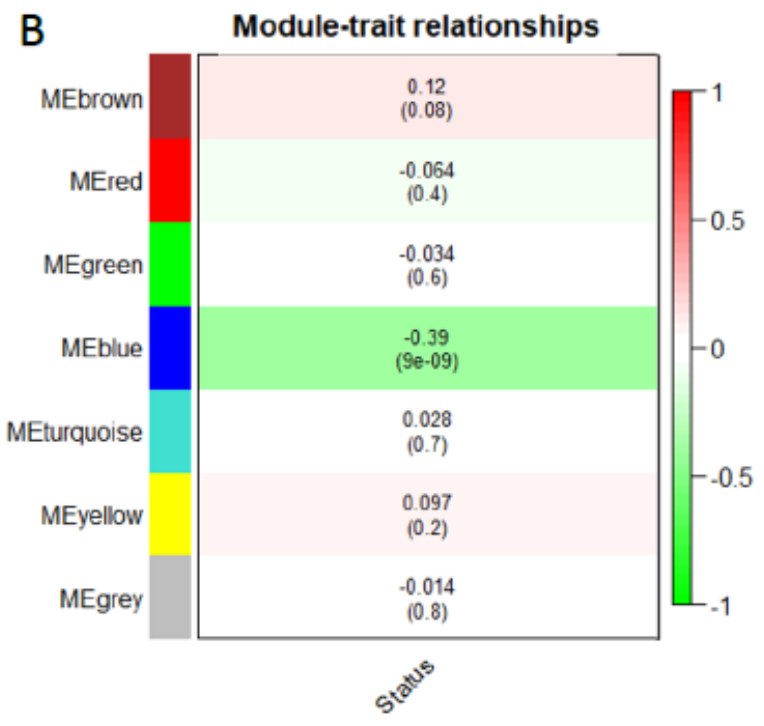

\section{Figure 3}

Network construction for GSE93987 using WGCNA. (A) Clustering dendrogram of samples based on their Euclidean distance. (B) Module-trait associations. Each row corresponds to a module eigengene, column to a trait. Each cell contains the corresponding correlation and p-value. The table is color-coded by correlation according to the color legend. (C) A scatterplot of Gene Significance (GS) for weight vs. Module Membership (MM) in the blue module. 

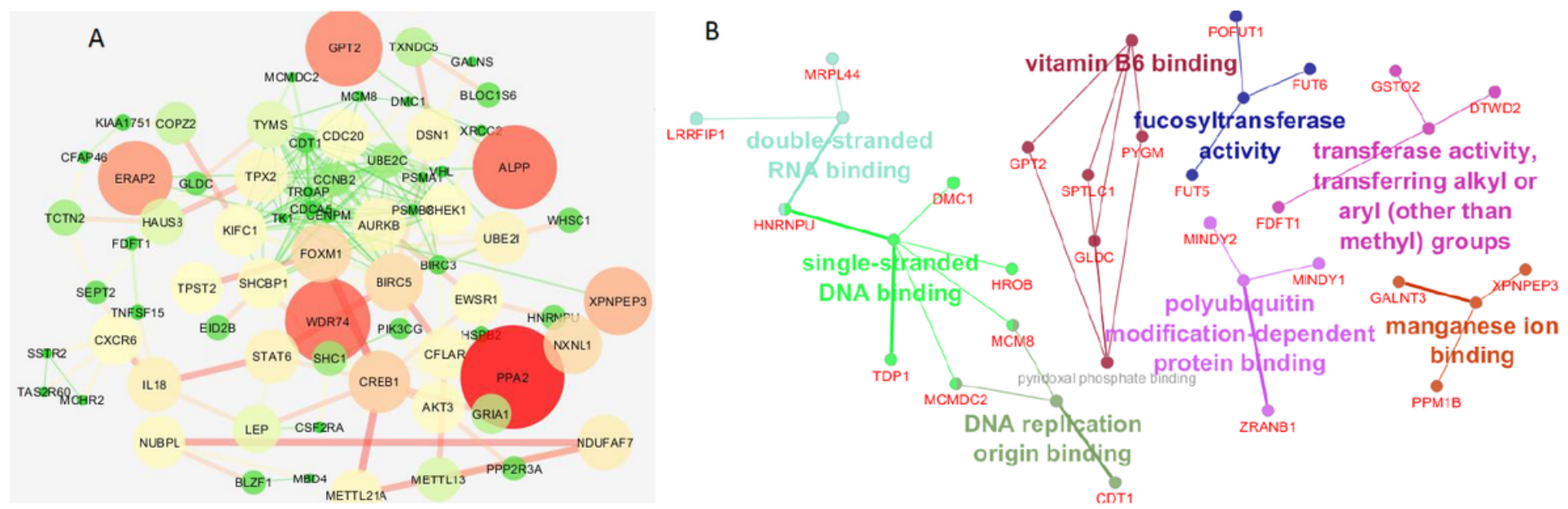

\section{Figure 4}

Enrichment of gens of lightcyan module. (A) PPI of genes based of their degree and central betweeness using STRING and cytoscape. (B) Identification of dysregulated pathways using CluGO plugin based on Cytoscape software. 


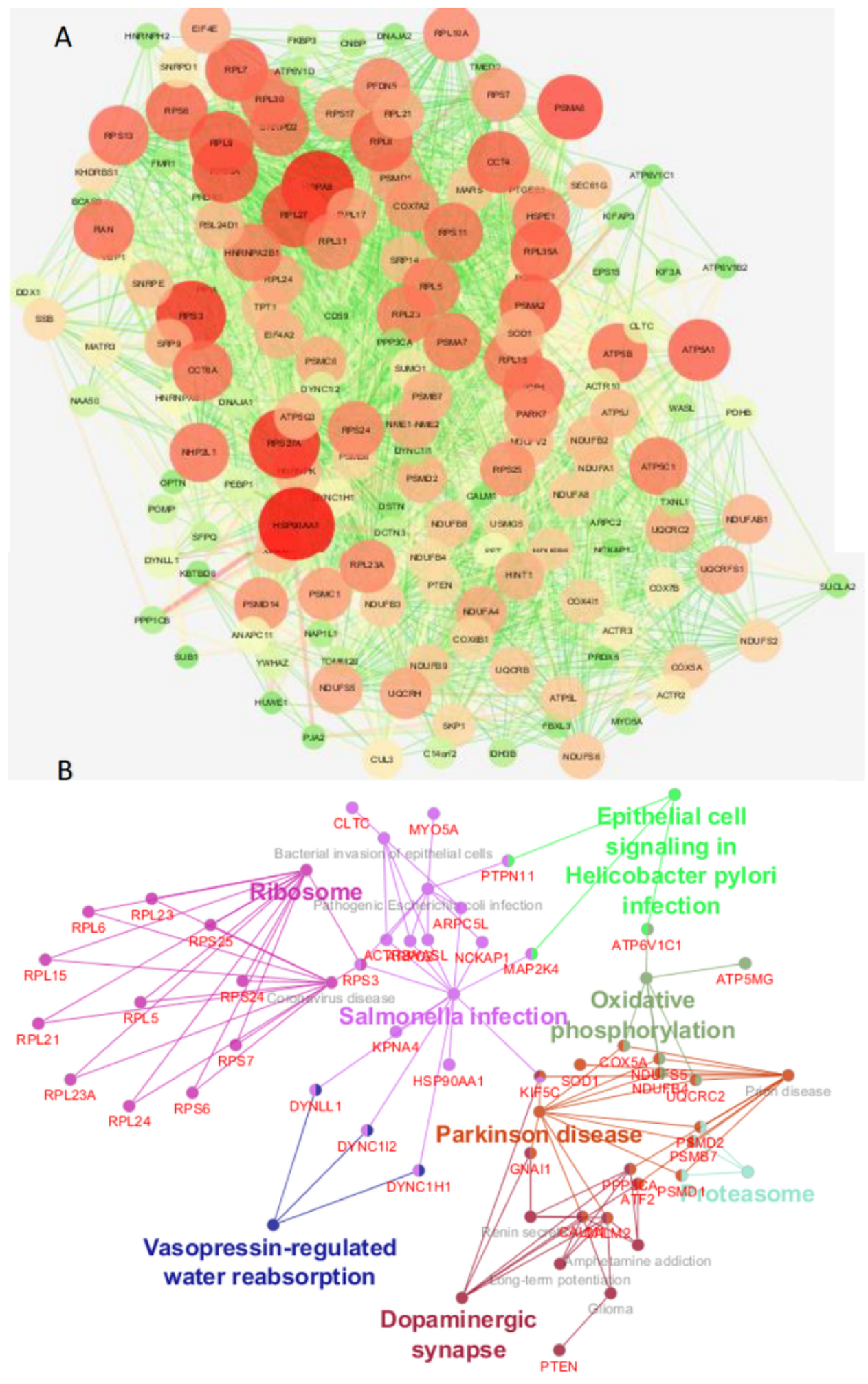

\section{Figure 5}

Enrichment of gens of blue module. (A) PPI of genes based of their degree and central betweeness using STRING and cytoscape. (B) Identification of dysregulated pathways using CluGO plugin based on Cytoscape software. 


\section{GSE54914}

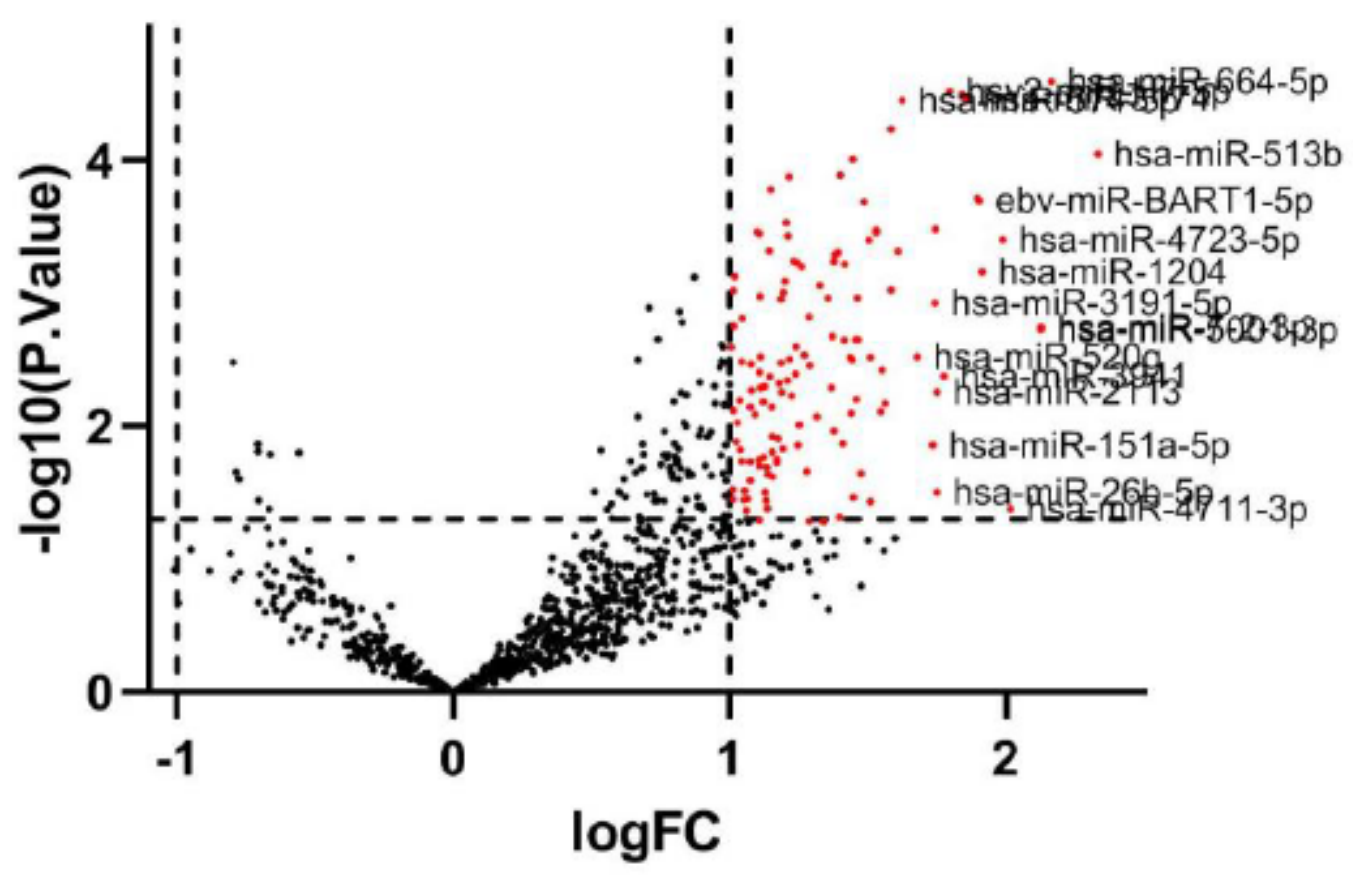

Figure 6

Volcano plot display of differentially expressed miRNAs between schizophrenia and healthy controls.

Every point in the plot represents a miRNA. Red points indicate miRNAs that are upregulated, blue points indicate miRNAs that are downregulated. 


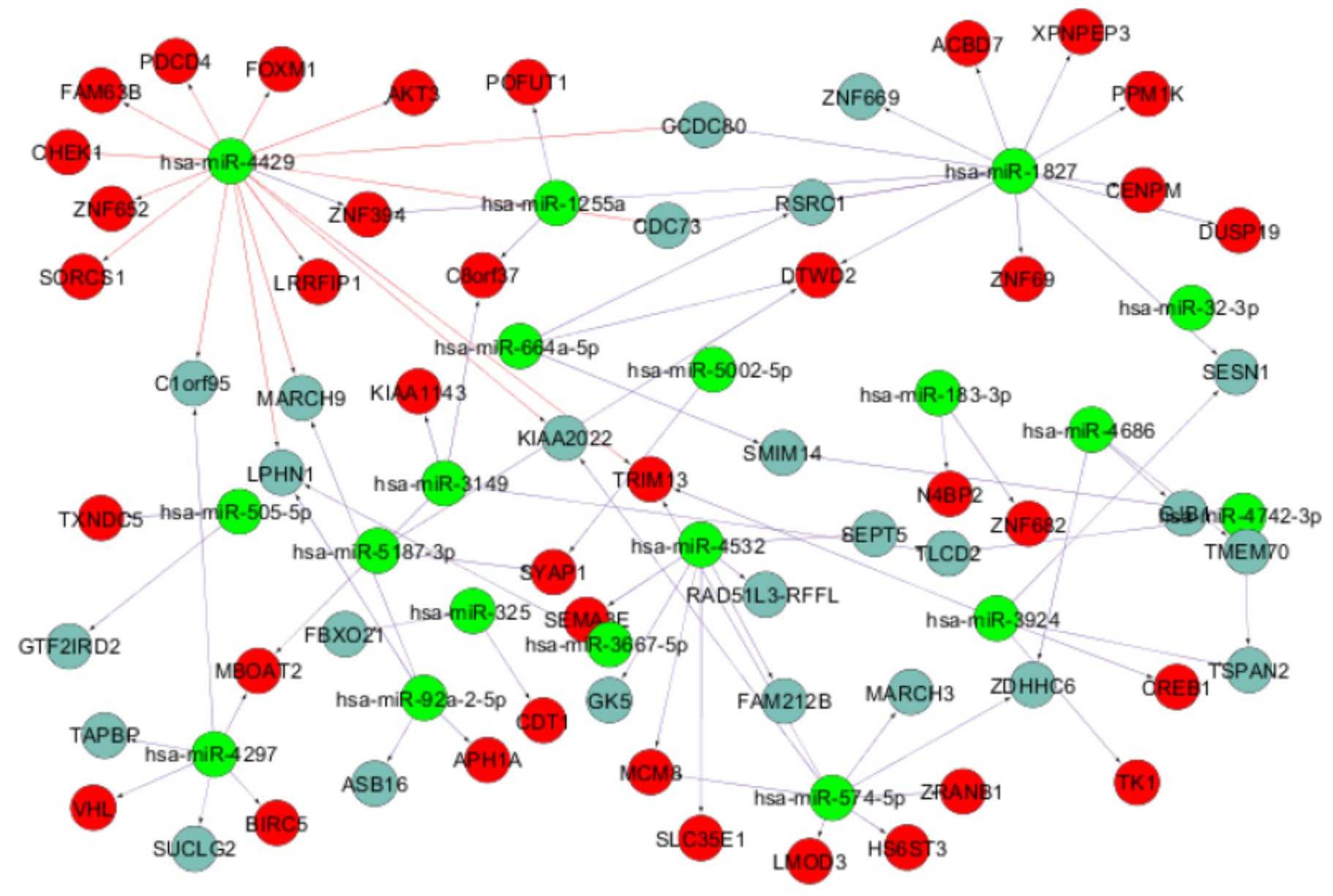

\section{Figure 7}

mRNA-miRNA regulatory network between DEMs and DEGs related to lightcyan module genes. Every node represents a gene or a miRNA, and each edge represents the interaction between genes and miRNAs. The colours red and blue represents genes, and green represents miRNAs. 


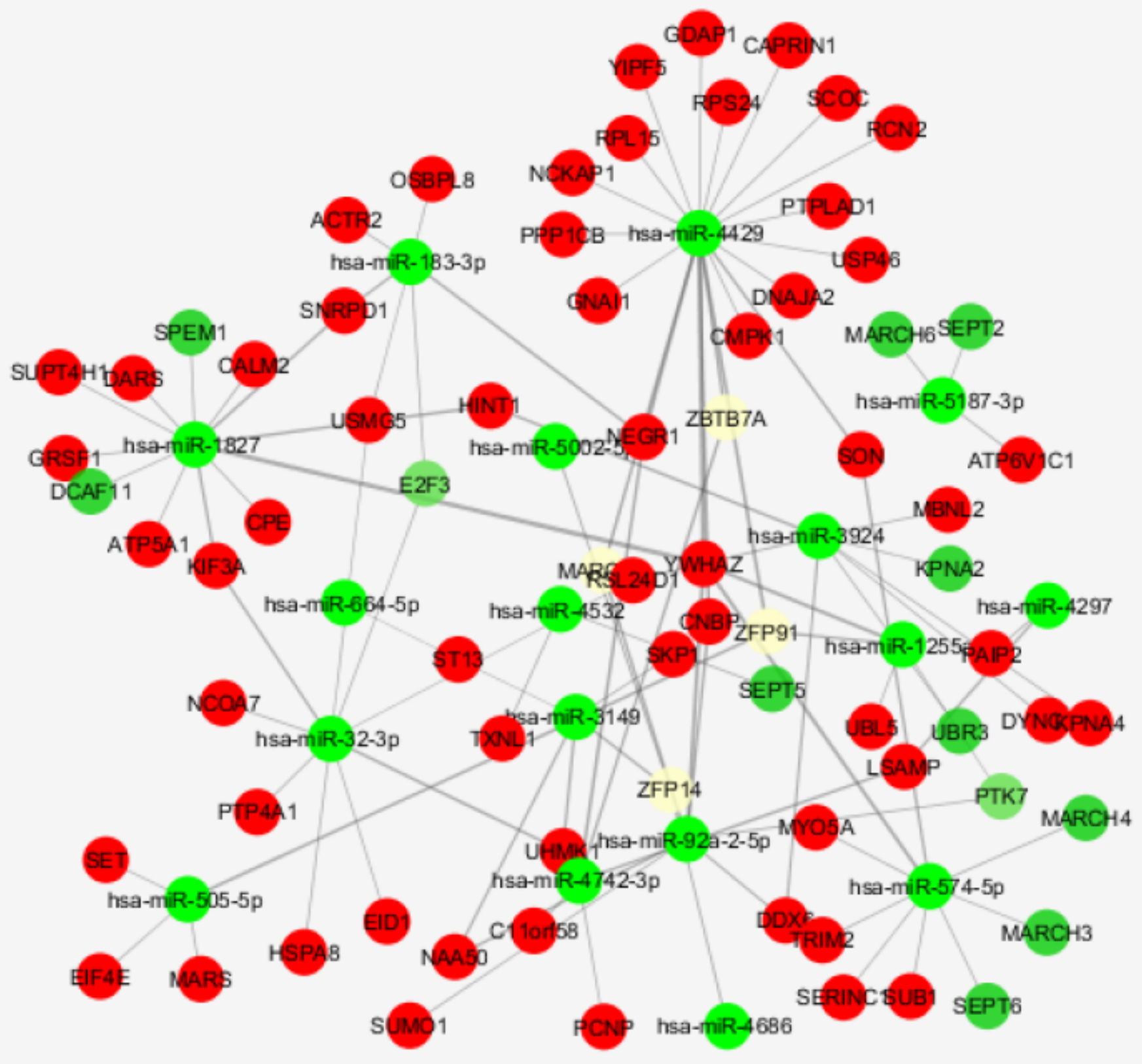

Figure 8

mRNA-miRNA regulatory network between DEMs and DEGs related to blue module genes. Every node represents a gene or a miRNA, and each edge represents the interaction between genes and miRNAs. The colours red represents genes, and green represents miRNAs. 


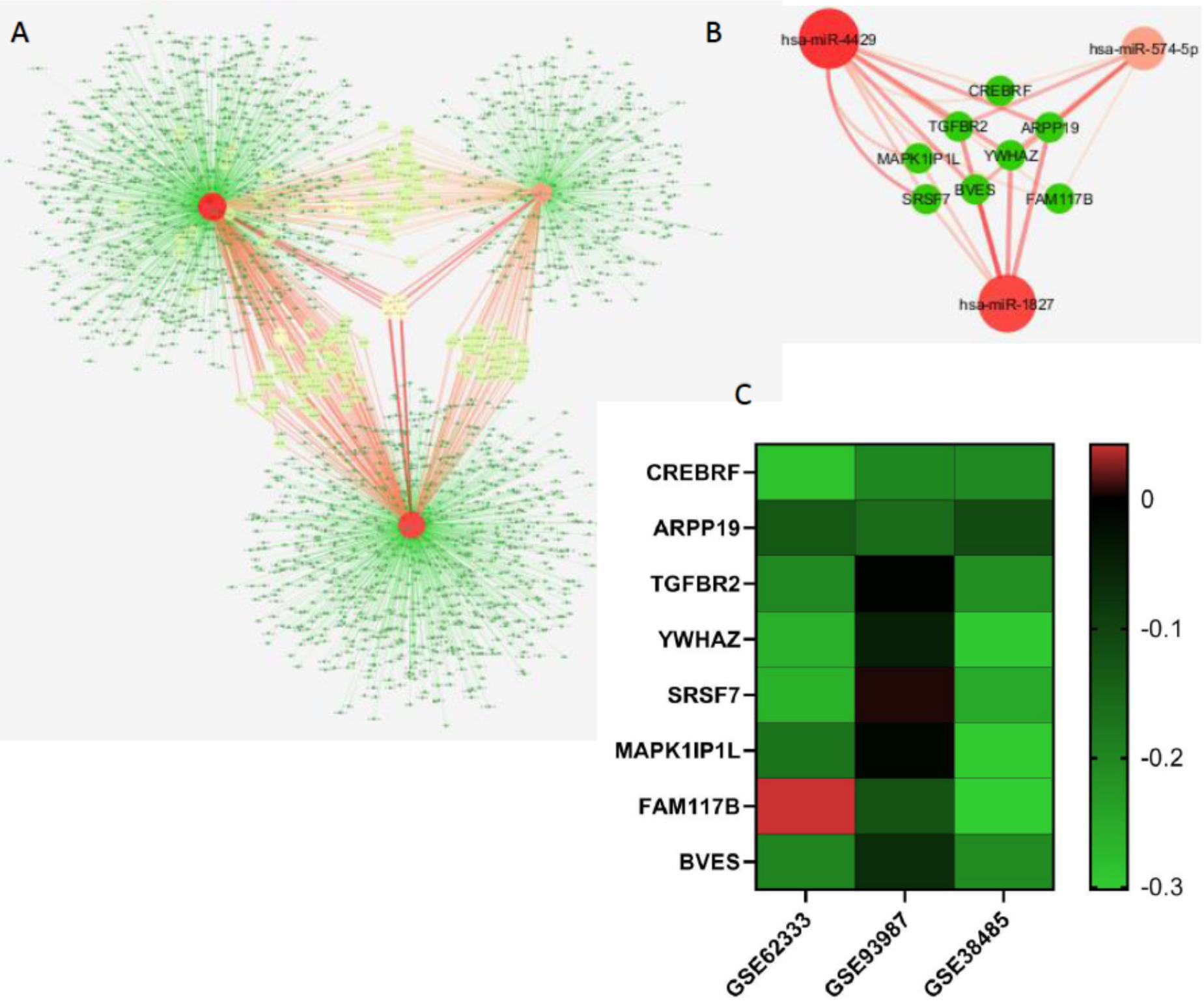

\section{Figure 9}

MiRNA-Targets interactions of the hub miRNAs including Has-miR-4429, Has-miR-1827, and Has-miR574-5P. (A) Nodes between three big red nodes (Has-miR-4429, Has-miR-1827, and Has-miR-574-5P) are common target genes of them and were shown as yellow nodes. (B) Eight genes including CREBRF, ARPP19, TGFBR2, YWHAZ, SRSF7, MAPK1IP1L, FAM117B and BVES are common target genes of three mentioned hub genes. (C) The expression status of these common target genes evaluated in three independent expression arrays (GSE62333, GSE93987, and GSE38485) and the results are shown as heatmap. 


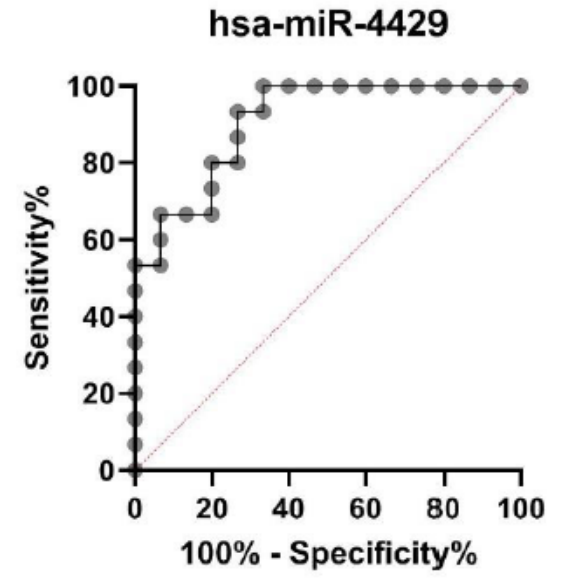

$\begin{array}{cc}\text { Area under the ROC curve } & \\ \text { Area } & 0.9067 \\ \text { Std. Error } & 0.05216 \\ \text { 95\% confidence interval } & 0.8044 \text { to } 1.000 \\ \text { P value } & 0.0001\end{array}$
hsa-miR-1827
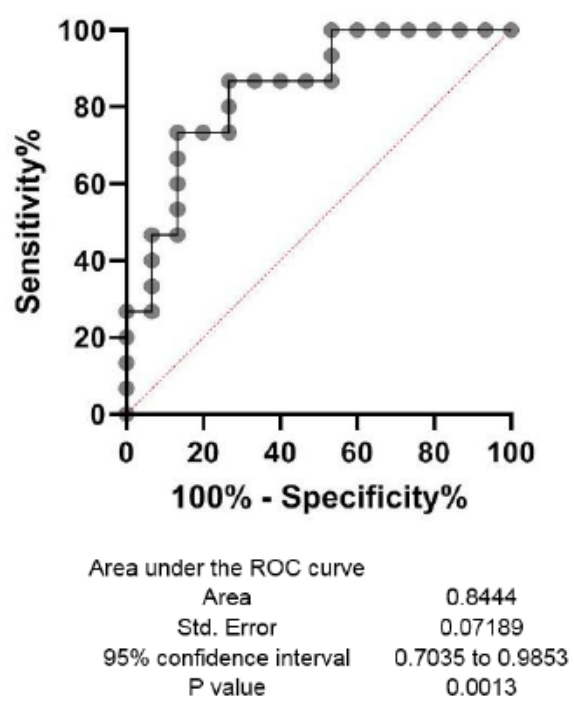

hsa-miR-574-5p

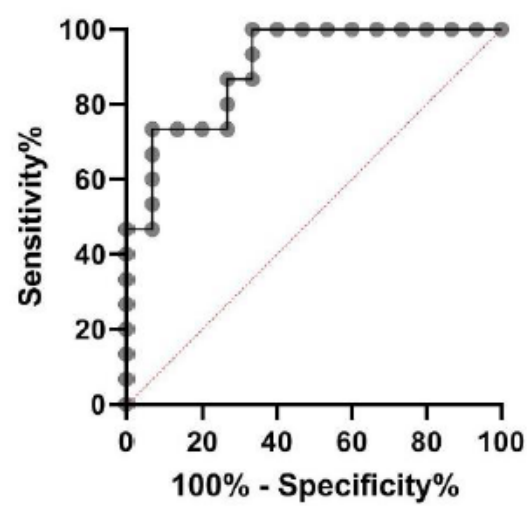

Area under the ROC curve

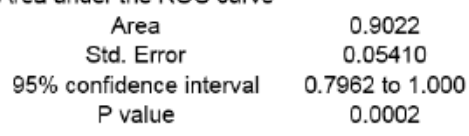

Figure 10

ROC curve analysis of three core miRNAs based on GSE54914 dataset. Receiver operating characteristic (ROC) curve and area under the curve (AUC) statistics are used to evaluate the capacity to discriminate schizophrenia from healthy controls with excellent specificity and sensitivity.
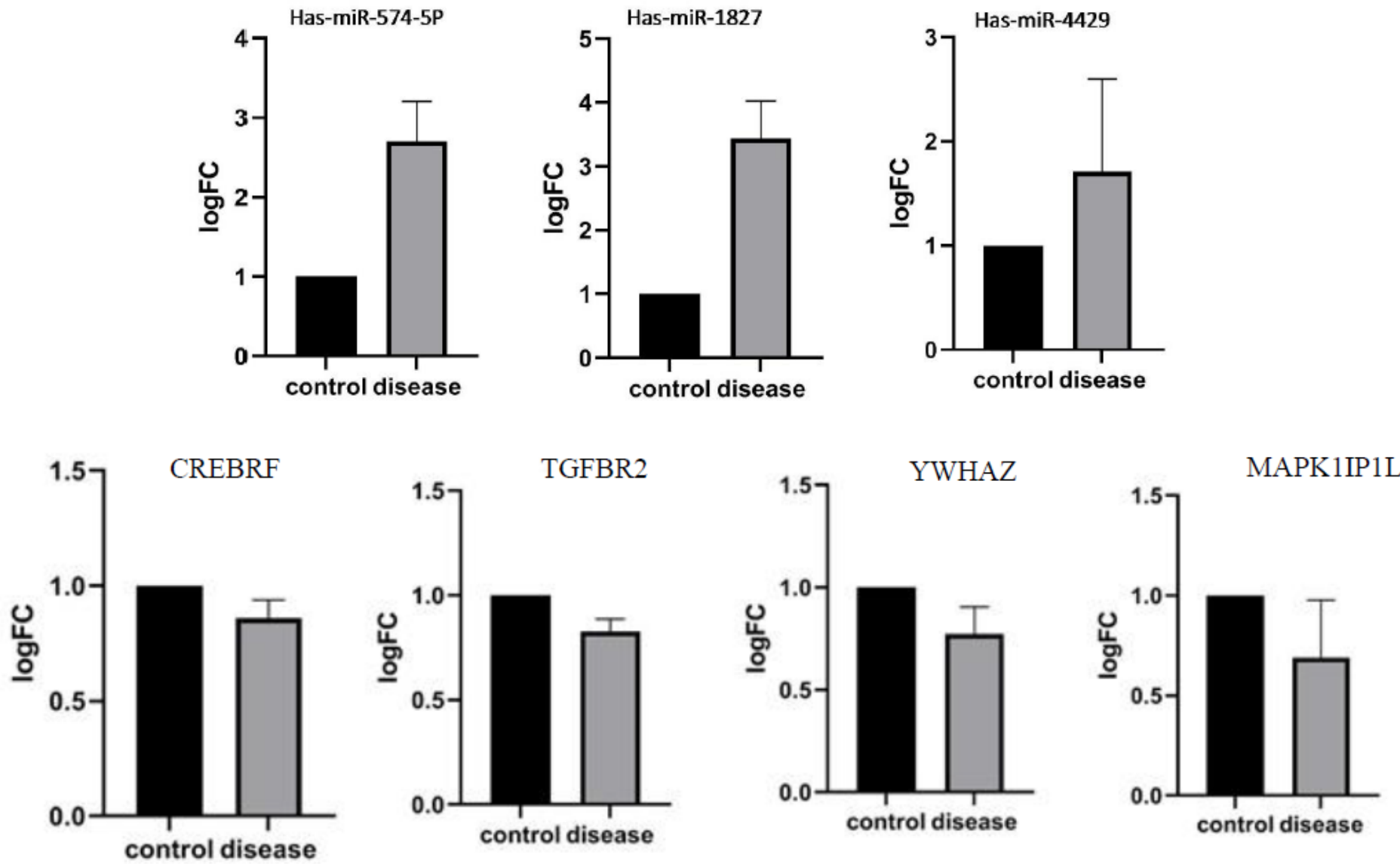


\section{Figure 11}

Real time PCR analysis for miRNAs and mRNAs for schizophrenia in comparison with healthy controls in blood samples. Bar diagram shows fold change in expression of core miRNAs. Statistical comparisons were made with respective $5 s$ rRNA for miRNAs and GAPDH for mRNAs. $\mathrm{P}<0.05$ for all genes and miRNAs.

\section{Supplementary Files}

This is a list of supplementary files associated with this preprint. Click to download.

- GraphicalAbstract.png 